Submitted for Publication in Chao, H-K., J. Reiss and S-T. Chen (eds), Philosophy of Science in Practice: Nancy Cartwright and the Nature of Scientific Reasoning. New York: Springer. (Penultimate version - To be revised.)

\title{
On Component Forces in Physics: A Pragmatic View
}

\author{
Darrell P. Rowbottom
}

Lingnan University

darrellrowbottom@ln.edu.hk

Do component forces exist? I argue that the answer lies in the affirmative, on historical and operational grounds.

\section{Introduction - Laws of Motion Vs. Force Laws}

One of Cartwright's (1980, 1983: ch. 3) most interesting and controversial claims is that component forces do not exist. ${ }^{1}$ A simple way to motivate this claim is to appeal directly to Newton's second law of motion, which is often presented as $\mathbf{F}=\frac{d \mathbf{p}}{d t} \cdot{ }^{2}$ If there is no change in a body's momentum, then there is no force acting on it. That is, provided that there are no forces of magnitude zero (and hence that a value of zero for

\footnotetext{
${ }^{1}$ Papers addressing the issue, or closely related issues to do with causality, include Creary (1981), Bigelow et al. (1988), Corry (2006), A. Wilson (2009), Spurrett (2001), J. Wilson (2009), Massin (2009), and Schrenk (2011).

${ }^{2}$ We will later touch on whether it is reasonable to ascribe this law to Newton. I use a momentum representation because this is an easy way to link classical mechanics with quantum mechanics and relativistic mechanics. In short, this definition of force has been retained, although the definition of momentum has changed. (In the former case, force and momentum are operators rather than vectors. In the latter case, momentum is defined in terms of relativistic mass.)
} 
$\mathbf{F}$ indicates the absence of any force). ${ }^{3}$ Now add that so-called 'component forces' may 'act' on a body without altering that body's momentum - consider, for example, the component force 'exerted' on you by the chair you sit on as you read - and it follows that not all component forces are really forces (even if 'component force' refers); $\neg \forall x(C x \rightarrow F x)$, where $F$ represents 'is a force' and $C$ represents 'is a component force'. Add also that in all cases where multiple component forces 'act', none is individually responsible for a rate of change of momentum equal to that specified in the second law, and it follows that they are not forces with some special property; $\forall x(F x \rightarrow \neg C x)$. (That is, on the further assumption that there are no situations where a single component force acts in isolation. This is uncontroversial on a straightforward understanding of 'component'.) It doesn't follow that there is no kind of entity other than a force - or a body - that's picked out by talk of 'component forces'; $\neg \exists C x$ is not a consequence of any of the prior reasoning. But it's possible that so-called 'component forces' are mere mathematical constructs; and this is the possibility that Cartwright $(1980,1983)$ seeks to exploit.

There is a mirror argument, however, which begins by instead considering so-called 'force laws', such as Newton's law of gravitation and Coulomb's law:

\footnotetext{
${ }^{3}$ Massin (Forthcoming: §4.3) argues for this conclusion. Moreover, Blay (2001) understands Newton’s verbal precursor to the modern version of the second law to state a sufficient condition. We would expect the modern version to follow suit in this respect (and therefore for an impulse on a body to be sufficient to change the body's momentum). We will discuss Newton's formulation in more detail in due course.

4 'Act' is in scare quotes because its use is metaphorical. The talk is of representations in a class of physical models.
} 


$$
\begin{gathered}
\mathbf{F}_{12}=-G \frac{m_{1} m_{2}}{r^{2}} \hat{\mathbf{r}}_{12} \\
\mathbf{F}_{12}=\frac{q_{1} q_{2}}{4 \pi \varepsilon_{0} r^{2}} \hat{\mathbf{r}}_{12}
\end{gathered}
$$

Since both laws may operate simultaneously, it is evident that there are possible scenarios where the two force vectors will cancel out (if summed). ${ }^{5}$ Two bodies with appropriate charges and masses will each have two equal and opposite forces acting on them, for any positive finite distance that they are apart. This appears to be what the laws say, irrespective of how such forces relate to motion. Indeed, we can even imagine weird possible worlds where such equal and opposite forces cause motion (or particular forms thereof). Hence, we can legitimately take the forces referred to in such 'force laws' as basic, or fundamental, and deny the existence of any further forces.

We have a standoff.

\section{Reading Equations: A Simple Mistake?}

Many physicists would respond to the previous section by declaring that both of the previous arguments are based on an elementary misunderstanding. This is as follows. $\mathbf{F}$ refers to net or resultant force in one equation, namely $\mathbf{F}=\frac{d \mathbf{p}}{d t}$, whereas $\mathbf{F}$ refers to

\footnotetext{
${ }^{5}$ I use these two laws for presentational reasons, e.g. to enable easier quotation of Cartwright (1983) at later points in the paper; it's possible, of course, to consider three bodies and just one of these laws. For instance, two of the bodies could exert equal but opposite attractive forces on the third.
} 
component forces in the others. ${ }^{6}$ In some cases, hypothetically at least, these different variables may take the same value. And the value is often the same for practical purposes. Consider, for instance, the motion of an ice hockey puck fired over a rink by a spring-loaded gun. Hooke's law $-\mathbf{F}=-k \mathbf{x}$, where $k$ is a constant for the specific spring in use - suffices in order to find a value for $\mathbf{F}$ to 'plug in' into the motion equation in order to predict the puck's maximum momentum: $\Delta \mathbf{p} \approx-k \mathbf{x}$. That is, even though Hooke's law is itself a rather crude approximation.

However, almost all authors on the existence of component forces agree that $\mathbf{F}$ represents two different variables in the aforementioned equations. (Wilson (2009) might think otherwise, judging by one of her comments, as we'll in the next section. ${ }^{7}$ ) And admitting this does not alter the thrust of the two opposing arguments presented in the previous section, which were highly simplified for presentational purposes. One approach is to begin with the law (or laws) of action, and to take this (or these) as definitional of 'force'. The other is to begin with the so-called 'force laws', and to take these as definitional of 'force'. ${ }^{8}$ Either way, it's possible for two parties to adopt different definitions of 'force' that are (superficially, at the bare minimum) grounded in classical mechanics.

I discuss definitions because I take it that almost all professional physicists concur that "Forces exist" and can competently perform numerous calculations using the

\footnotetext{
${ }^{6}$ See also Massin (2009: $\left.§ 1.2\right)$.

${ }^{7}$ All subsequent uses of 'Wilson' refer to Jessica Wilson.

${ }^{8}$ There's also a third way, which is to take both kinds of law to have definitional relevance. I think that this is the correct view, as will become apparent.
} 
aforementioned equations, but that these facts mask underlying disagreements. These disagreements concern how the equations should be physically interpreted, and what exists as a result. Some will take component forces to exist. Others won't. Others still will not have given any thought to, and will be neutral on, or even disdainfully disinterested in, such interpretative matters. My own view is that this state of affairs is reasonable, in so far as it's possible to avoid such commitments while maintaining the ability to save the phenomena in many domains in an economical fashion, although I shan't say any more on this issue here. ${ }^{9}$ My aim in this section is to emphasize that the success of physics - and a semblance of complete consensus therein on the issue of forces in a classical context - doesn't depend on there being an explicitly or implicitly agreed ontological story about resultant and component forces. ${ }^{10}$

\section{Cartwright vs. Creary on Forces}

There are only four logically possible positions on the existence of component forces and resultant forces:

\footnotetext{
${ }^{9}$ From my pragmatic perspective, very roughly, mechanics is primarily about understanding how to arrange objects such that they stay still or move in particular ways. Derivatively, this gives understanding of how objects will behave when in particular arrangements. For my more detailed views on scientific progress, see Rowbottom (In Press) and Rowbottom (Manuscript).

${ }^{10}$ This is one sense in which Kuhn's (1996) emphasis on shared exemplars (of puzzle solving), within a discipline, is important; see Bird (2004) and Rowbottom (2011: §3) for more on this. Any physicist worth her salt will have cut her teeth by solving many problems in elementary mechanics involving the equations cited previously, as well as derivative constant acceleration equations. Even elementary twodimensional problems solved before vector formulations are learned, e.g. involving projectile motion, involve resolving motion into independent perpendicular components.
} 
(i) Only resultant forces exist.

(ii) Only component forces exist.

(iii) Both resultant forces and component forces exist.

(iv) Neither resultant forces nor component forces exist.

Exceptionally strong reasons to think that (iv) is false - exceptionally strong, that is, relative to the available reasons for thinking that any one of the other possibilities obtains - will appear in the course of the subsequent discussion. They are primarily experiential in character. So three options remain.

Cartwright (1980: 78) champions (i) with explicit reference to the process of vector addition:

The vector addition story is, I admit, a nice one. But it is just a metaphor. We add forces (or the numbers that represent forces) when we do calculations. Nature does not "add forces". For the "component" forces are not there, in any but a metaphorical sense, to be added... [T] he force of size $\frac{G m m^{\prime}}{r^{2}}$ and the force of size $\frac{q q^{\prime}}{r^{2}}$ [sic] are not real, occurrent forces. In interaction, a single force occurs - the force we call the "resultant" - and this force is neither the force due to gravity not the electric force.

Wilson (2009: 529-530) perceptively points out the following, however. First, Cartwright (1980) assumes that resultant forces exist, and so only sees fit to argue 
against (iii). Second, Cartwright's argument against (iii) is not general, in so far as she only undermines one way to interpret 'the vector addition story' literally. Nonetheless, a reasonable argument, presented by Creary (1981) and developed further by Wilson (2009: §3.2) concerns the threat of causal overdetermination. Wilson (2009: 539-540) summarizes this as follows:

The resultant force is, in itself, sufficient to bring about the resulting motion... But the distinct component forces, each acting during the time in question, also appear sufficient to bring about the effect: this is what the superposition principle (grounding the appropriateness of calculating the resultant force via vector addition, and relatedly, of explanation by composition of causes) tells us... [But] surely the effect in a given case of conjoined circumstances is not caused twice over - one by the component forces assumed to be present, and again by the (dffierent) [sic] resultant force assumed to be present.

For present purposes, I propose to take this argument as decisive; (iii) is therefore out. But what of (ii)? Creary (1981: 152) outlines it as follows:

Newton's second law of motion is interpreted as saying that the set of all natural forces acting on a body will produce an acceleration in the direction of the mathematical resultant of the forces which is directly proportional to the magnitude of the resultant, and inversely proportional to the mass of the body. Thus, the law implies that multiple natural forces acting together will have the same net effect as would a single natural force having the magnitude and direction of their vector sum. Due to the nature of vector addition, this further 
implies that multiple temporally extended forces (i.e., impulses) acting simultaneously will have the same net effect as they would if they acted separately and consecutively... I do not grant the physical reality of overall resultant forces. ${ }^{11}$

I think that this is the correct view, and I will defend it in the remainder of the piece, by championing an operational (yet partial) definition of force that I take to fit with the historical development of the concept. I will offer my historical remarks first, in order to motivate the definition.

Before I do so, however, I want to address the following recent objection to this view, which is presented by Wilson (2009: 534-535):

If the interpretation were correct, we would expect either that (a) standard formulations of the law would indicate that the law takes multiple forces, not a single force, as input (e.g., as $\sum \mathbf{F}_{\mathbf{i}}=m \mathbf{a}$ rather than $\mathbf{F}=m \mathbf{a}$ ), or that (b) standard presentations of the law as usually formulated would indicate that the seemingly single force $\mathbf{F}$ at issue is a merely technical innovation introduced to simplify treatment of multiple component forces (along lines, e.g., of the standard disclaimer in presentations of laws appealing to the center of mass of a system, which may be located where nothing is). Since standard

\footnotetext{
${ }^{11}$ Not all component forces are natural. I also agree with Creary (1981: 151-152) that:

A distinction is made between natural component forces, which arise directly from the action of various real physical causes, and mathematical component forces, which arise merely from the artificial resolution of vectors, and thus lack physical existence.
} 
formulations and presentations of the second law (starting with Newton, and continuing to the present day) typically do not conform to either (a) or (b), Creary's interpretation is correspondingly implausible. (Wilson 2009: 534535)

This line of argument is weak. First and foremost, formulae in physics are not typically presented in such a way that their standard interpretation, let alone their proper interpretation, may easily be 'read off them'. Rather, equations demand only of their users that they can work out the correct values to input, in order to get the desired output. In this regard, consider how Coulomb's law contains a factor of $4 \pi$, whereas Newton's law of gravitation does not. But the presence of an expression representing the surface area of a sphere, i.e. $4 \pi r^{2}$ (in the non-vector version of the law), suggests a field interpretation, by way of analogy with equations governing intensity. Think of the light emitted by a point source, and of concentric hollow spheres, to see the point. Double your distance away from the source, and the intensity is quartered. And note that this difference between Coulomb's and Newton's law is a matter of convention - it stems from the different way that fluxes are defined in gravitational and electromagnetic contexts, as is evident from Gauss's laws although this is doubtless unknown to most users of said equations.

If this still seems implausible, consider that inertial mass and gravitational mass are also conceptually distinct; hence, $m$ in the second law of motion for any given body might be found, experimentally, to differ in value from $m$ in the law of gravitation. 
More precisely, any body may be said to have two distinct properties, $m_{\text {in }}$ and $m_{\text {grav }}$; and the fact that these take the same value for all bodies does not entail, although it might strongly suggest, that they are the same. I suppose Wilson might respond that most physicists know about this, through learning about the equivalence principle. One the one hand, however, even many of the most talented physics students will not have recognized (or seen the need for) this distinction before being exposed to general relativity. On the other, similar conceptual distinctions pass more easily unnoticed. Consider the difference between active and passive gravitational mass, discussed by Bondi (1957) and Jammer (1961, 2008), for example. ${ }^{12}$ I doubt that many contemporary physicists are aware of, let alone care about, this.

Second, a formulation of the second law in terms of $\Sigma$ notation can be found in at least one popular university physics textbook. (I have not surveyed enough textbooks to comment on how typical this is. Note, however, that Wilson (2009) provides no evidence in support of her claim that this is atypical.) Cutnell and Johnson (2012: 88) introduce the second law as follows:

Often, several forces act on an object simultaneously... In such cases, it is the net force, or the vector sum of all the forces acting, that is important. Mathematically, the net force is written as $\sum \overrightarrow{\mathbf{F}}$, where the Greek capital letter... denotes the vector sum. Newton's second law states that the acceleration is proportional to the net force acting on the object...

\footnotetext{
${ }^{12}$ In classical mechanics, it is a consequence of the third law that these have the same value. But the distinction is not easy to spot, because the third law is expressed in terms of forces. We'll return to this distinction in relation to the third law a little later, in section five.
} 


$$
\sum \overrightarrow{\mathbf{F}}=m \overrightarrow{\mathbf{a}}
$$

One can also find introductory web pages where the same formulation appears, e.g. http://www.livescience.com/46560-newton-second-law.html and http://physics.info/newton-second/. (The second page, from 'The Physics Hypertextbook', also declares that the equation 'is often written with net force as the subject'.) Furthermore, there are other similar resources where the second law is explicitly formulated in terms of a different symbol for net force, such as $\mathrm{F}_{\text {net }}$; see, for instance, $\quad$ http://www.physicsclassroom.com/class/newtlaws/Lesson-3/Newton-sSecond-Law. And if net force is 'the vector sum of all the forces acting', as suggested above, then this is an equivalent formulation.

I would add that Wilson's use of 'starting with Newton' is inappropriate, because modern 'standard presentations' differ from his in several notable respects. If one insists on linking 'force' in modern physics with Newton's presentation thereof, for instance, then it is extremely dubious - as Dijksterhuis (1961), Jammer (1961), Cohen (1970), and Blay (2001) argue - that $\mathbf{F}=\frac{d \mathbf{p}}{d t}$ is an appropriate equation to be discussing. In short, this is because Newton did not mean 'rate of change of momentum' by 'alteration of motion' (in his definition of the second law). The law was not presented in differential form until 1716; this was in Hermann's Phoronomia. And when Euler (1750: 195-196) subsequently presented a formula (or more accurately formulae) resembling this, he claimed to have made a new discovery. 
Thus, Wilson's initial objection to Creary's interpretation fails. However, Wilson (2009: 535) subsequently offers a stronger argument that resultant forces exist, which is experiential in character:

We experience forces in interacting with the world, which are directly associated with our accelerations; and these forces are frequently associated with multiple influences. In the latter cases, we always seem to experience resultant forces (whether or not we also experience component forces): forces associated with a single magnitude and direction, that directly result in our accelerations.

I will counter this argument later, in section five.

\section{A Brief History of Force}

I propose to take it for granted that Newton's conception of force - or more pertinently, as noted above, the conception of force that developed in the century or so thereafter - differed from any previous conception in some respects. (Mach (1893), Bridgman (1927) and Gillies (1972), among other authors who consider the history of mechanics, agree on this.) But it does not follow that said conception was not an extension of a considerably older concept of force, rather than an entirely novel notion. And in case it was an extension, we should ask, carefully, whether component forces - or a close analogue to these - appear in the older concept. 
Mechanics began with statics. One of the earliest surviving works is On the Equilibrium of Planes, by Archimedes of Syracuse (287-212 B.C.), in which the law of the lever is derived. Mach (1893: 9-10) presents the two assumptions on which the derivation is founded as follows:

a. Magnitudes of equal weight acting at equal distances (from their point of support) are in equilibrium.

b. Magnitudes of equal weight acting at unequal distances (from their point of support) are not in equilibrium, but the one acting at the greater distance sinks.

Mach continues by arguing that these axioms are not 'self-evident', in so far as defending them involves numerous theoretical assumptions, especially concerning the irrelevance of other factors (such as other properties of the bodies and the presence of observers). Of special interest, however, is the assumption that: 'weights but also their distances from the supporting point... are circumstances determinative of motion.' (Mach 1893: 9-10) We now talk of moments, which Mach refers to as 'statical moments'. He identifies Da Vinci (1452-1519) as 'the first to recognize the importance of the general notion'. However, he opines that 'the way that really led to this idea is not now fully ascertainable' (Mach 1893: 21).

So there was a concept of weight distinct from statical moment, such that it is a crucial factor determining whether equilibrium obtains in systems of bodies, in ancient times. And this was developed somewhat by Stevinus (1548-1620), who 
employed the principle of the parallelogram of forces (without, as Mach (1893: 33) explains, 'explicitly formulating it'). Mach (1893: 35-36) suggests that Stevinus knew that it was true, but not why it was true, on the basis of a reconstruction of Stevinus's reasoning process. But even if this humble claim is correct, it illustrates that some kind of notion of component weight, at the bare minimum, predates Newton.

However, Stevinus's work is also remarkable in one further respect, which is that he considers tension in strings, as well as weight; see Mach (1893: 31) for a brief presentation of some of his findings. ${ }^{13}$ Thus, I conclude that there is a notion of component force, grounded in statics, which predates Newton. Gillies (1972) agrees, even if his use of 'but' downplays the significance of this: 'We must admit that in the study of Statics and Equilibrium a notion of force had evolved, but it was little more than a slight generalization of the idea of weight.' Gillies (1972) also quotes the following passage, in which Mach (1893: 57) opines that the old concept was generalized, not entirely replaced, in subsequent dynamics:

Previous to Newton a force was almost universally conceived simply as the pull or the pressure of a heavy body. The mechanical researchers of this period dealt almost exclusively with heavy bodies. When... in the Newtonian epoch, the generalization of the idea of force was effected, all mechanical principles

\footnotetext{
${ }^{13}$ Stevinus is perhaps best known for his ingenious derivation of the principle of the inclined plane. For a presentation and discussion of his thought experiment, see Rowbottom (2014). As Mach (1892: 23) points out, however, the principle of the inclined plane can be derived from the principle of the lever (although I think this derivation depends on thinking of tensions and weights as both being kinds of force).
} 
known to be applicable to heavy bodies could be transferred at once to any forces whatsoever. It was possible to replace every force by the pull of a heavy body on a string.

\section{An Operational Account of Force}

There is also a reasonable operational account of force that fits with the brief history of the concept described above; and I take this to have been provided by Bridgman (1927). But before I present and develop this, I will explain the significance that I take such an operational account to have.

It is well known that Bridgman expressed an extreme view concerning meaning in his early work: 'In general, we mean by any concept nothing more than a set of operations; the concept is synonymous with the corresponding set of operations.' (Bridgman 1927: 5). And he took this principle to hold not only in physics, but also more broadly: 'It would doubtless conduce greatly to clarity of thought if the operational mode of thinking were adopted in all fields of inquiry as well as in the physical.' (Bridgman 1927: 30). The problems with this extreme view are also reasonably well-known, and are illustrated by Lindsay (1927), Gillies (1972), and Chang (2009). Most importantly, it is presently uncontroversial - in philosophy, at least - that there are meaningful concepts that cannot be operationally defined. And it is not my intent to swim against the tide of opinion on this matter. It does not follow, however, that operational definitions are irrelevant to meaning. I hold instead, roughly, that the presence of an operational definition is an indicator of meaning. 
I will now make this claim more precise, with closer reference to Bridgman (1927). First, let's restrict the scope of our discussion to (phenomena-relevant) terms introduced in the natural sciences. Second, let's address meaning, which is a slippery and controversial notion in the philosophy of language. Lewis (1970: 19) helpfully distinguishes between two distinct projects which have been associated with 'meaning': 'the description of possible languages or grammars as abstract semantic systems whereby symbols are associated with aspects of the world... and... the description of the psychological and sociological facts whereby a particular one of these abstract semantic systems is the one used by a person or population'. Bridgman was concerned mainly with the former project. In particular, he was concerned with whether concepts appropriately connect with reality. He wrote:

$[\mathrm{O}]$ ne must inquire into the meaning of the concepts as used by the questioner, and it will often be found that these concepts can be defined only in terms of fictitious properties, as Newton's absolute time was defined by its properties, so that the meaning to be ascribed to the question in this way has no connection with reality. [my emphasis] (Bridgman 1927: 29)

We need not take 'connects with reality' to be metaphysics-laden, e.g. to presuppose some kind of transcendental realism. 'Connects with the phenomena' will serve as a suitable substitute, given that the scope of the present discussion is the natural sciences. 
Third let's consider what counts as a meaningful (or phenomena-relevant) question on Bridgman's view. This is apt in so far as the overarching concern of this paper is to answer, 'Do component forces exist?' Bridgman (1927: 28) advocated the following principle:

\section{Question Operationalism (QO)}

'If a specific question has meaning, [then] it must be possible to find operations by which an answer may be given to it. ${ }^{, 15}$

I reject this. But I propose, to a first approximation, that a converse principle is reasonable:

\section{Converse Question Operationalism (CQO)}

If it is possible to find operations by which an answer may be given to a question, then the question has meaning.

The modal nature of this statement is, however, potentially troublesome. Strictly speaking, for example, it allows that a question can have meaning despite this being presently indeterminable. The verification principle of logical positivism encounters similar difficulties, e.g. in the version proposed by Ayer (1936), in so far as this concerns that which is verifiable. This cannot mean 'possible to verify in practice',

\footnotetext{
15 Bridgman (1927: 28) mentioned expressions too, and implicitly endorses a similar principle concerning these. Appeal to concepts may be required in order to extend this principle to cover these. For example, Bridgman presumably intended that an expression is meaningless if it involves at least one meaningless concept; and the same for questions and statements, mutatis mutandis.
} 
because it is evident that a claim such as 'The far side of the moon is heavily cratered' was meaningful long before technological means by which to view the far side of the moon were available. (It is hardly as if the creation of Luna 3 - or failing that, the successful design thereof - rendered the claim meaningful! ${ }^{16}$ ) But if 'verifiable' means 'possible to verify in principle', then it is impossible (in many cases, at least) to determine what is, and isn't verifiable. (Or if one takes a sufficiently liberal view about how the possible overlaps with the conceivable, every well-formed sentence will prove to be verifiable.)

We may therefore improve the previous claim by excising the modal element:

\section{Converse Question Operationalism (CQO*)}

If there are operations by which an answer may be given to a question, then the question has meaning.

This principle does not require that a question possesses meaning only in so far as it may be answered by some available (or known) operations. Thus, we need not require an operational definition to be exhaustive; rather it can be partial and incomplete. Since such definitions need not be exhaustive, it follows that they need not consist of necessary and sufficient conditions (for membership of the relevant set of things). And reflection on how difficult it is to provide satisfactory intensional definitions even for simple everyday words, such as 'table' and 'chair', should lead one to appreciate that any expectation otherwise is unrealistic, whether the definition is

\footnotetext{
${ }^{16}$ The images from Luna 3 were rather indistinct, and could, at best, only very weakly verify such a statement on Ayer's view; Apollo 8 might be substituted, if this is a concern.
} 
operational or not! Nevertheless, it is possible to provide fully operational definitions that specify some sufficient conditions (for set membership). And this is how I believe we should understand the definition to follow.

This brings us to Bridgman's (1927: 102-103) operational definition of force, which I reproduce at some length:

In origin the concept doubtless arises from the muscular sensations of resistance experienced from external bodies. This crude concept may at once be put on a quantitative basis by substituting a spring balance for our muscles, or instead of the spring balance we may use any elastic body, and measure the force exerted by it in terms of its deformation... The concept of force so defined is limited to static systems; it is the task of statics to the find the relation between the forces in systems at rest. We next extend the force concept to systems not in equilibrium, in which there are accelerations... We here encounter a new concept, that of mass, which as it is originally met is entangled with the force concept... Suffice it to say that we are eventually able to give to each rigid material body a numerical tag characteristic of the body, such that the product of this number and the acceleration it receives under the action of any given force applied to it by a spring balance is numerically equal to the force, the force being defined, except for a correction, in terms of the deformation of the balance, exactly as it was in the static case. In particularly [sic], the relation found between mass, force, and acceleration applies to the spring balance itself by which the force is applied, so that a correction has to 
be applied for a diminution of the force exerted by the balance arising from its own acceleration.

We now extend the scope of our measurements... We extend the concept of force, and say that any body accelerated is acted on by a force, and the magnitude of this force is defined as that which would have been necessary to produce in the same body the same acceleration with a spring balance in empty space. There is physical justification for this extension in that we find we can remove the acceleration which a body acquires in a gravitational field by exerting on it with a spring balance a force of exactly the specified amount in the opposite direction. This extended idea of force may also be applied to systems in which there are electrical actions.

Three aspects of this passage are especially noteworthy. First, the operational definition of 'force' is grounded in statics, in line with the history of the concept. So when Bridgman writes that mass 'was originally entangled with the force concept', he refers, inter alia, to the role that weight played in early statics - e.g. in the principle of the lever and the principle of the inclined plane - in line with our discussion of the history of mechanics in the previous section. Second, the operational definition may simply be extended - and need not be radically changed - in order to bear on dynamics. In effect, this is by considering the potential for a coiled spring (or appropriately similar body) to generate motion in other bodies. Consider using a specific test body, such as a small pebble. There is a one-to-one correspondence between each possible deformation state of a given spring (in a fixed orientation) and 
how it will cause the pebble to move when released. (That is, provided that the initial conditions concerning the pebble are the same, and the surrounding environment is (relevantly) similar. ${ }^{17}$.) Third, the relationship between spring deformation states and changes in motion is bidirectional. With reference again to our test pebble, there is also a one-to-one correspondence between each possible motion state and how it will cause a given spring (in a fixed orientation) to deform. ${ }^{18}$ (That is, with similar provisos as before. For simplicity's sake, in comprehending this point, it's helpful to imagine that the spring is aligned appropriately with the pebble when they make contact; that is, to think in one dimension. But one could consider the deformation of a different elastic entity, such as a sponge, if desired.)

A nice addition to Bridgman's discussion is to think about how two spring balances (of the same type) interact in a uniform gravitational field. Imagine each balance has a locking switch, which prevents its spring from deforming, and consider the following. The two balances are connected, and held vertically. The bottom balance is locked, whereas the top balance is unlocked. A reading is taken from the top balance, which has value $r$. This represents the force of the bottom balance on the top balance. The top balance is now locked, and the bottom balance is unlocked. A reading is taken

\footnotetext{
${ }^{17}$ Bridgman imagines a laboratory in distant space, in order to finesse some of these difficulties. But this is not required. Or if it is, this difficulty is in no way peculiar to the concept of force. There are no closed systems, strictly speaking, although physics often proceeds, in its definitions and derivations, as if there are.

${ }^{18}$ Naturally, the motion may be retarded by the use of multiple springs instead. This fits with the idea, discussed below, that whether multiple component forces are present may, in some situations, be difficult or impossible to determine.
} 
from the bottom balance. This represents the force of the top balance on the bottom balance. It is seen to have value $r$. Both balances are unlocked, as a final step, and seen to read value $r$. The third law is corroborated.

Now let's turn our attention to component forces and resultant forces. Again, we may think in terms of multiple spring balances. Consider first the scenario depicted in figure one, where two balances of the same type are locked in the same state of compression, but are perpendicularly oriented, and in contact with a solid cylinder. (If you're fussy, you can imagine this happening in a zero-g environment, in a vacuum.)

\section{Fig. 1 - Cylinder with Two 'Locked' and Compressed Spring Balances}

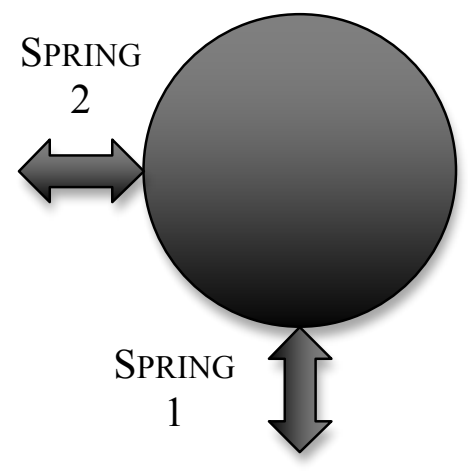

When the balances are simultaneously unlocked, the cylinder will follow a specific trajectory. This may be recorded. The system may then be reset, and a third spring balance, oriented at forty-five degree angle to the previous two (in the plane of the diagram), may be added. See fig. 2. Through experimentation, it may be determined that this third balance can be deformed such that the cylinder remains in equilibrium when all the balances are unlocked simultaneously.

Fig. 2 - Static Cylinder Acted on by Three 'Unlocked' Spring Balances 


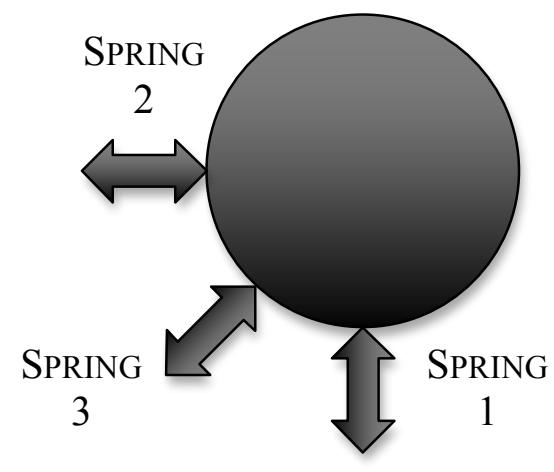

Now the system may be reset for a final time, and the initial two balances removed. It may be determined that when the third spring acts equally in the opposite direction to that in which it acted in the previous scenario, this results in the same cylinder trajectory as that recorded in the first. (The 'third balance' - i.e., 'spring three' - will be extended in the second scenario, depicted in fig. 2, and begin compressed in the third scenario.)

Let us now think about which forces can be said to exist in the above scenarios. I take the answer to be simple. There are forces when are where there are spring balances in states of deformation. So there are two forces present in figure one, and three forces present in figure two. (When the balances 'locked', the forces are acting on the locking mechanism. When they're 'unlocked', they're acting on the cylinder.) That's all there is to say on the matter.

We are now in a position to revisit Wilson's (2009: 535-536) experiential argument for the existence of resultant forces, which I will now reproduce at greater length: 
We experience forces in interacting with the world, which are directly associated with our accelerations; and these forces are frequently associated with multiple influences. In the latter cases, we always seem to experience resultant forces (whether or not we also experience component forces): forces associated with a single magnitude and direction, that directly result in our accelerations... [I]n at least some cases where multiple influences are at issue, we seem to experience resultant forces without experiencing the associated component forces. For example, when one rides in a tilt-a-whirl, one feels only a single force pinning one to the wall, not two distinct forces, one gravitational and one centrifugal... Experience thus provides us with good reason to posit resultant forces as existing in conjoined circumstances.

The response to this, on the basis of the prior operational definition of force, is as follows. We have discovered, through our empirical investigations, that multiple forces cannot in general be distinguished from single forces, although it is sometimes clear that they are present. A simple and direct way to show this is with the following thought experiment. Consider two spring-loaded boxing gloves, each of which punches as hard as the other (as one might test extensively, experientially, by submitting oneself to a comprehensive clobbering). However, one boxing glove is powered by a lone spring, whereas the other boxing glove is powered by two springs in parallel. ${ }^{19}$ Thus one force powers one glove, whereas two forces of the same type but of lesser magnitude power the second. But the only way to determine this is to examine the mechanism (unless one spring breaks, or some such). Being punched

\footnotetext{
${ }^{19}$ It's conceivable, of course, for there to be $n$ parallel springs having the same effect, for any integer $n$, subject to the appropriate physical constraints on spring size being obeyed.
} 
repeatedly will not help, no matter how entertaining it might be for onlookers. (It is also remarkable that Wilson mentions centrifugal force, which is a pseudoforce according to the standard view in physics. If we experience pseudoforces - as we presumably would when on the inside surface of a spinning hollow cylinder in a zerog environment, given Wilson's account - then so much the worse for the experiential argument. Our experiences are presumably not to be trusted, at least in a significant class of reference frames. ${ }^{20}$ )

We have seen that on the operational definition provided above, component forces exist in some circumstances in which resultant forces do not. And I rest content with this conclusion. We have seen nevertheless that in some scenarios, it is difficult, or even impossible, to determine precisely which component forces are acting. But this is unremarkable; measurement has limitations in any area of physics.

\section{Conclusion}

On an operational (partial) definition of force that is sensitive to the historical roots of the contemporary concept, some component forces exist.

\footnotetext{
${ }^{20}$ I don't think we 'experience' forces in the way that Wilson appears to think we do. I contend instead that that the observation statements we take to be true concerning our bodily sensations are theoryladen, and that they theories we use are inclined to employ are often false (e.g., folk physical theories). (It might be added that it's possible to argue that we can experience accelerations, but not forces.)
} 


\section{Acknowledgements}

Thanks to Martin Hardcastle, Olivier Massin, and Julian Reiss for comments on an earlier version of this piece. This research was supported by the UGC, Hong Kong ('The Instrument of Science', Humanities and Social Sciences Prestigious Fellowship).

\section{References}

Ayer, A. J. 1936. Language, Truth and Logic. London: Victor Gollancz.

Bigelow, J., B. Ellis, and R. Pargetter. 1988. 'Forces', Philosophy of Science 55, 614630.

Bird, A. 2004. 'Thomas Kuhn', in E. N. Zalta (ed.), The Stanford Encyclopedia of Philosophy. URL: http://plato.stanford.edu/archives/fall2004/entries/thomas-kuhn/.

Blay, M. 2001. 'Force, Continuity and the Mathematization of Motion at the End of the Seventeenth Century', in J. Z. Buchwald and I. B. Cohen (eds), Isaac Newton's Natural Philosophy, pp. 225-248. Cambridge, MA: MIT Press.

Bondi, H. 1957. 'Negative Mass in General Relativity', Reviews of Modern Physics $29,423$.

Cartwright, N. 1980. 'Do the Laws of Physics State the Facts?', Pacific Philosophical Quarterly 61, 75-84.

Cartwright, N. 1983. How the Laws of Physics Lie. Oxford: Oxford University Press. 
Chang, H. 2009. 'Operationalism', in E. N. Zalta (ed.), Stanford Encyclopedia of

Philosophy. URL: http://plato.stanford.edu/archives/fall2009/entries/operationalism/.

Cohen, I. B. 1970. 'Newton's Second Law and the Concept of Force in the Principia', in R. Palter (ed.), The Annus Mirabilis of Sir Isaac Newton: 1666-1966, pp 143-185. Cambridge, MA: MIT Press.

Corry, R. 2006. 'Causal Realism and the Laws of Nature', Philosophy of Science 73, $261-276$.

Creary, L. 1981. 'Causal Explanation and the Reality of Natural Component Forces', Pacific Philosophical Quarterly 62, 148-157.

Cutnell, J. D. and K. W. Johnson. 2012. Physics. (9 ${ }^{\text {th }}$ Ed.)

Dijksterhuis, E. J. 1961. The Mechanization of the World Picture. London: Oxford University Press.

Gillies, D. A. 1972. 'Operationalism', Synthese 25, 1-24.

Jammer, M. 1961. Concepts of Mass in Classical and Modern Physics. Cambridge, MA: Harvard University Press.

Jammer, M. 2008. Concepts of Mass in Contemporary Physics and Philosophy.

Princeton: Princeton University Press.

Kuhn, T. S. 1996. The Structure of Scientific Revolutions. Chicago: University of Chicago Press ( $3^{\text {rd }}$ Ed. $)$.

Lewis, D. 1970. 'General Semantics', Synthese 22, 18-67.

Lindsay, R. B. 1937. 'A Critique of Operationalism in Physics', Philosophy of 
Science 4, 456-470.

Mach, E. 1893. The Science of Mechanics: A Critical and Historical Account of Its

Development. Chicago, IL: Open Court. (4 ${ }^{\text {th }}$ Ed., 1919).

Massin, O. 2009. 'The Metaphysics of Forces', Dialectica 63, 555-589.

Massin, O. Forthcoming. 'The Composition of Forces', British Journal for the Philosophy of Science.

Rowbottom, D. P. 2011. 'Stances and Paradigms: A Reflection', Synthese 178, 111119.

Rowbottom, D. P. 2014. 'Intuitions in Science: Thought Experiments as Argument Pumps, in A. R. Booth and D. P. Rowbottom (eds), Intuitions, pp. 119-134. Oxford: Oxford University Press.

Rowbottom, D. P. In Press. 'Scientific Progress without Increasing Verisimilitude: In Response to Niiniluoto', Studies in History and Philosophy of Science. DOI:

10.1016/j.shpsa.2015.01.003

Rowbottom, D. P. Manuscript. The Instrument of Science.

Schrenk, M. 2011. 'Interfering with Nomological Necessity', Philosophical Quarterly $61,577-597$.

Spurrett, D. 2001, 'Cartwright on Laws and Composition', International Studies in the Philosophy of Science 15, 253-268.

Wilson, A. 2009, 'Disposition-manifestations and Reference-frames', Dialectica 63, $591-601$. 
Wilson, J. 2009. 'The Causal Argument Against Component Forces', Dialectica 63, 525-554. 